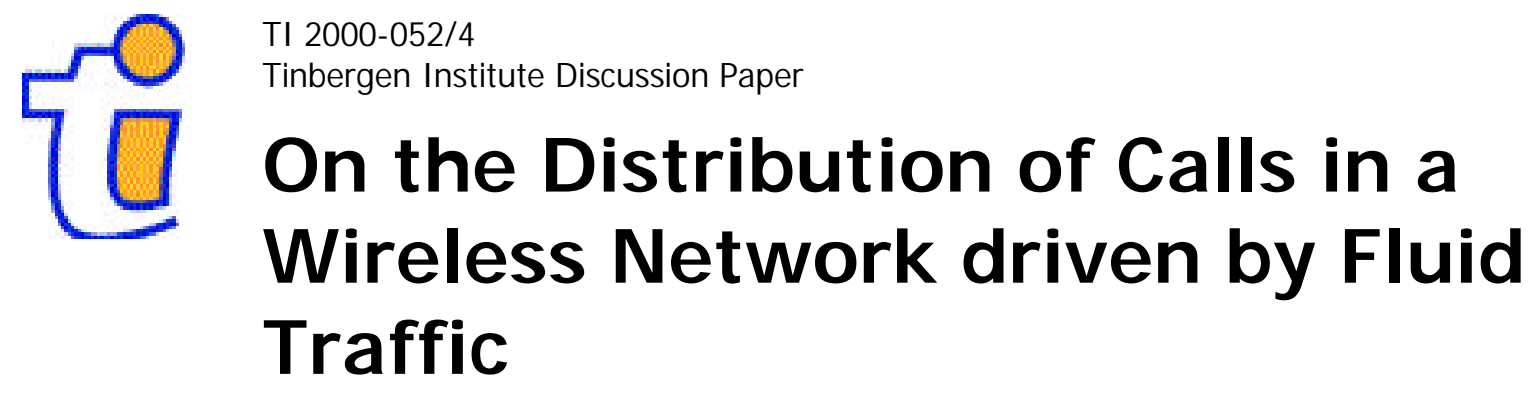

Aljaž Ule

Richard J. Boucherie 
Tinbergen Institute

The Tinbergen Institute is the institute for economic research of the Erasmus Universiteit Rotterdam, Universiteit van Amsterdam and

Vrije Universiteit Amsterdam.

Tinbergen I nstitute Amsterdam

Keizersgracht 482

1017 EG Amsterdam

The Netherlands

Tel.: +31.(0)20.5513500

Fax: $\quad+31 .(0) 20.5513555$

Tinbergen I nstitute Rotterdam

Burg. Oudlaan 50

3062 PA Rotterdam

The Netherlands

Tel.: $\quad+31 .(0) 10.4088900$

Fax: $\quad+31 .(0) 10.4089031$

Most TI discussion papers can be downloaded at

http://www.tinbergen.nl 


\title{
On the distribution of calls in a wireless network driven by fluid traffic
}

\author{
Aljaž Ule* Richard J. Boucherie ${ }^{\dagger}$ \\ Department of Operations Research, Universiteit van Amsterdam, \\ Roetersstraat 11, 1018 WB Amsterdam, The Netherlands \\ \{ule,boucheri\}@fee.uva.nl \\ July 6,2000
}

\begin{abstract}
This note develops a modelling approach for wireless networks driven by fluid traffic models. Introducing traffic sets that follow movement of subscribers, the wireless network with time-varying rates is transformed into a stationary network at these traffic sets, which yields that the distribution of calls over the cells of the network depends on the call length distribution only through its mean. The result is extended to a network of infinite server queues with time-varying arrival rates.
\end{abstract}

Keywords: Telecommunications, Traffic, Stochastic processes

\section{Introduction}

Wireless communications has been a rapidly growing service in the field of telecommunications. In contrast with this rapid growth, the capacity of wireless networks is severely restricted causing service degradation due to e.g. blocking or interruption of calls. Due to the cellular nature of wireless networks, these networks have been modelled using queueing networks; a cell is represented as an Erlang loss queue, where the limited capacity of the wireless network is represented by the limited number of servers. Calls moving among the cells (handovers) are modelled as customers routing among the queues, see Massey \& Whitt (1994).

The representation of a wireless network using the queueing network formulation is only partly valid, since (i) the exact location of a call on e.g. a road is required for interference, and (ii) in a cellular network the call length is related to the call, whereas the service time of a customer in a queueing network is related to the queue. When a call moves from one cell to another, the residual call length must

${ }^{*}$ Corresponding author.

${ }^{\dagger}$ The research of R.J. Boucherie is supported by the Technology Foundation STW, applied science division of NWO and the technology programme of the Ministry of Economic Affairs, The Netherlands. 
be taken into account. Only under the assumption of exponentially distributed call lengths and holding times in the cells a standard queueing network formulation is justified as in this case the call length can be resampled upon a handover due to the memoryless property of the exponential distribution, see Everitt (1994). For general call lengths this is no longer correct as we have to explicitly deal with the residual call length of calls entering a cell, and for general holding times in the cells we have to explicitly take the location of the calls into account. This note investigates the effect of the call length distribution on the transient distribution of calls over the cells of the network, and presents sufficient conditions for the call length to affect the distribution of calls over the cells only through its mean.

A queueing model of a wireless network with calls extending over multiple cells was presented in Boucherie \& Van Dijk (2000), where the network was characterized by the call length distribution and the call holding times in the cells. In equilibrium, the influence of the call length distribution on the distribution of the calls over the network was investigated. This note extends these results to networks with nonstationary arrival rates to the cells generated by a non-stationary Poisson process with rate determined by the density of subscribers in that cell.

First, to determine the exact location of subscribers, a fluid traffic model for subscriber mobility is investigated in detail. Rather than considering the location of subscribers on the location space $\Omega$, e.g. a road or a set of roads, we consider the volume, $\Phi_{t}$, under the density curve over this location space at time $t$. Subscribers are assumed to be randomly placed at locations inside this volume. Obviously, the projection of these user locations on $\Omega$ results in the distribution of subscribers over $\Omega$ at time $t$. We characterize (mobility of) subscribers via (movement of) subsets $\phi \subset \Phi_{t}$, referred to as traffic sets. Due to our modelling assumptions, that are shown to include standard traffic models, subscribers follow the path of the traffic sets.

Second, under the realistic assumption that calls follow the mobility of subscribers, the influence of the call length distribution is investigated. Here the traffic sets play an important role: due to our transformation, the process counting the number of active calls in a traffic set is stochastically equivalent to a stationary infinite server queue, which yields insensitivity with respect to the call length distribution. This insensitivity result is shown to carry over to the distribution of calls over the cells of the network that obviously do have time-varying arrival rates.

The resulting model is closely related to the Poisson Arrival Location Model (PALM) introduced in Massey \& Whitt (1993). The general PALM is not directly amenable for performance analysis, and further specification is required to obtain tractable models. In Massey \& Whitt (1994) the PALM is applied to wireless networks, and in Leung et al. (1994) a fully Markovian setting is analyzed. In these references, in accordance with the queueing network approach, the distribution of the holding times in the cells is taken as the main distribution governing the behaviour of the network. In contrast, this note provides an alternative specification of the PALM investigating the influence of the call length distribution on the distribution of calls over the cells of the network. Via a transformation of the process on the location space $\Omega$ into the traffic sets, a straightforward analysis of the influence of the call length distribution can be provided. This demonstrates that our modelling approach is suitable for performance analysis of wireless networks.

Here is the organization of this note. Section 2 presents our modelling approach 


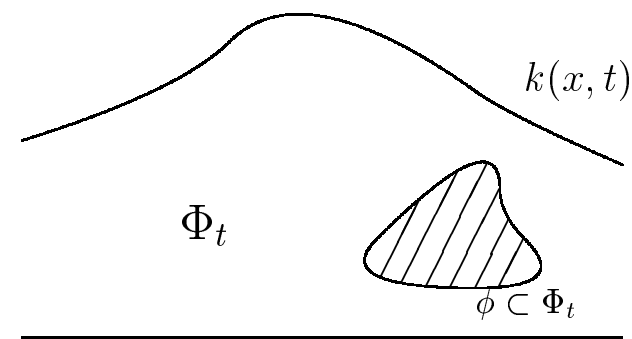

location space $\Omega$

(a)

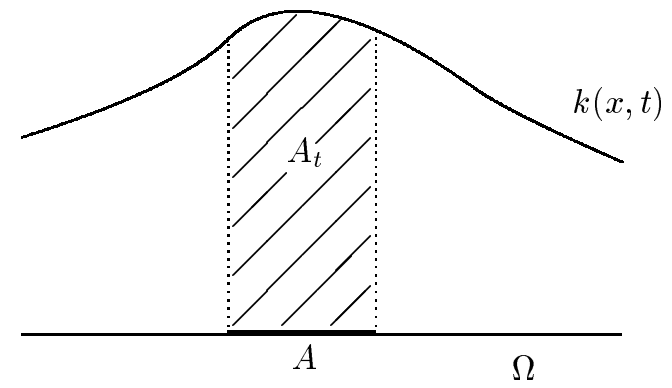

(b)

Figure 1: (a) Traffic $\Phi_{t}$ at time $t$. (b) Traffic in cell $A$ at time $t$.

for a network with deterministic subscriber mobility. Section 3 illustrates that this approach includes standard road traffic models, and extends the results to networks of queues with exponential holding times in the queues, but general call length distribution.

\section{Model and main result}

Consider a location space $\Omega \subset \mathbb{R}^{m}$. The distribution of subscribers over $\Omega$ is determined by the summable density $k: \Omega \times \mathbb{R} \rightarrow[0, \infty)$, i.e. $k(x, t)$ gives the mass of subscribers per unit space on location $x \in \Omega$ at time $t$. The density of subscribers changes in time according to a fluid traffic model. To this end, let

$$
\Phi_{t}:=\{(x, h) ; x \in \Omega, 0 \leq h \leq k(x, t)\} \subset \Omega \times \mathbb{R}
$$

be the traffic at time $t$, i.e. $\Phi_{t}$ denotes the set under the graph of the density curve $k(x, t)$ at time $t$ (see Fig. 1(a)). Movement of subscribers is modelled by movement of traffic sets $\phi \subset \Phi_{t}$. A point $\theta \in \Phi_{t}$ represents the infinitesimal traffic set including $\theta$. Of particular interest for wireless networks is the behaviour of the traffic volume in a cell $A \subset \Omega$. Therefore, for any $A \subset \Omega$ we introduce the traffic in cell $A$ at time $t$,

$$
A_{t}:=\{(x, h) ; x \in A, 0 \leq h \leq k(x, t)\} \subset \Phi_{t},
$$

and the traffic mass in cell $A$ at time $t$ (see Fig. 1(b))

$$
V\left(A_{t}\right)=\int_{A} k(x, t) d x
$$

Similarly, for each $\phi \subset \Phi_{t}$ we introduce the mass $V(\phi)$ as the Euclidean volume of $\phi$ :

$$
V(\phi)=\int_{\Phi_{t}} 1[x \in \phi] d x
$$

where $1[E]$ denotes the indicator function of event $E$. Movement of subscribers is determined by the traffic flow function

$$
L:\left\{(t, \theta, u) ; t \in \mathbb{R}, \theta \in \Phi_{t}, u \in \mathbb{R}\right\} \rightarrow \Omega \times \mathbb{R},
$$



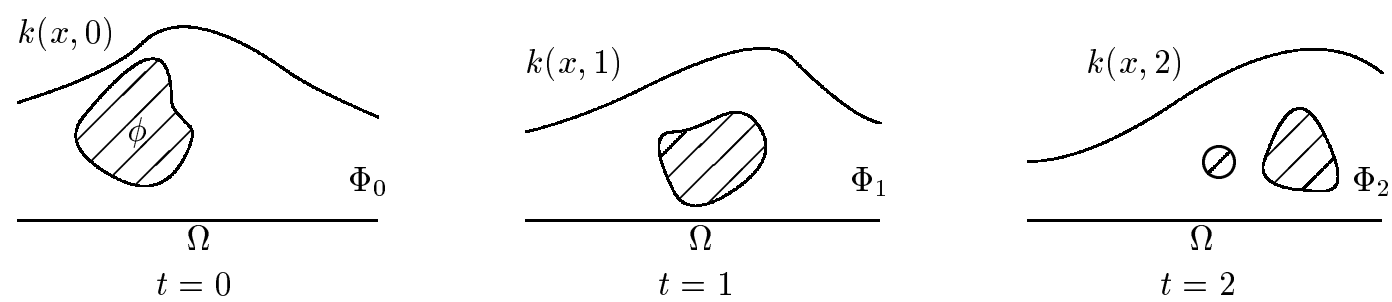

Figure 2: $L(0, \phi, t)$ describes the evolution of the traffic set $\phi \subset \Phi_{0}$.

where $L(t, \phi, u) \subset \Phi_{u}$ gives the position at time $u$ of the traffic set $\phi \subset \Phi_{t}$ (see Fig. 2 ). Assume that the traffic flow function has the following properties, for $t, s, u \in \mathbb{R}$,

L1. $L(t, \cdot, u): \Phi_{t} \rightarrow \Phi_{u}$ is a bijection,

L2. $L(s, L(t, \phi, s), u)=L(t, \phi, u)$ and $L(t, \phi, t)=\phi$ for all $\phi \subset \Phi_{t}$,

L3. $V(\phi)=V(L(t, \phi, u))$ for all $\phi \subset \Phi_{t}$, i.e. the traffic flow function is volume preserving.

The assumptions on the traffic flow function imply that the system is closed, i.e. external arrivals and departures of subscribers to the system are excluded. In particular, applying L1, L2 and L3, for each $t, u \in \mathbb{R}$,

$$
\int_{\Omega} k(x, t) d x=\int_{\Omega} k(x, u) d x .
$$

External arrivals and departures can be included in the model by labelling a subset of $\Omega$ as 'outside' and then consider subscribers moving to and from 'outside' as departures and arrivals to the system, respectively.

In this paper we are interested in the distribution of calls over the cells of the network. Subscribers are assumed to generate fresh calls at random times, independent of their location and movement, and independent of other calls. More subscribers generate more calls and here it is assumed that this relation is linear. Fresh calls are generated uniformly over traffic. For any $A \subset \Omega$, arrival times of fresh calls to cell $A$ form a Poisson process with non-stationary arrival rate

$$
\lambda_{A}(t):=\alpha \int_{A} k(x, t) d x=\alpha V\left(A_{t}\right),
$$

which is proportional to the density of subscribers in cell $A$ at time $t$, where $\alpha$ is the arrival rate of fresh calls per unit mass of subscribers. For notational convenience the results below are restricted to a constant unit mass arrival rate $\alpha$. As is indicated in Remark 2 below the results can readily be extended to a non-stationary arrival rate $\alpha(t)$. The Poisson process of call arrivals to cell $A$ corresponds to a Poisson arrival process to traffic sets $\left\{A_{t} ; t \in \mathbb{R}\right\}$ in which calls arriving to cell $A$ at time $t$ are uniformly distributed over $A_{t}$. The fresh call arrival processes for disjoint cells $A, B \subset \Omega$ are independent.

A call remains active during a generally distributed period of time (the call length) $S$ with distribution $G$ and finite first moment $\tau:=\mathbb{E}[S]$, independent of other calls, its location and generation time. A call generated in $\phi \subset \Phi_{t}$ at time $t$ will move 
along $\Omega$ according to the traffic flow function $L$, independent of its call length and other active calls: if a call was generated by a certain subscriber, it will follow the path of this subscriber for the whole period it is active.

For a call generated at time $s$ in $x \in \Phi_{t}$ we call the point $L(t, x, 0)$ its owner, i.e. it is the position of $x$ at time 0 . Due to L1 and L2, the path of each call through the system is uniquely determined by its arrival time, its owner and its call length. If a call is generated at time $t$ with owner $x$ and call length $\triangle t$, its path is a function $\ell:[t, t+\triangle t] \rightarrow \Omega \times[0, \infty), \ell(u)=L(0, x, u), t \leq u \leq t+\triangle t$. For each traffic set $\phi \subset \Phi_{t}$, let

$$
L^{\phi}:=\{(L(t, \phi, s), s) ; s \in \mathbb{R}\}
$$

be its trace through the system. A traffic set $\widehat{\phi}$ such that $(\widehat{\phi}, u) \in L^{\phi}$ will be called trace $L^{\phi}$ at time $u$. Two important properties of traces are embodied in the following lemma.

Lemma 1 For $\phi \subset \Phi_{t}$ and $\widehat{\phi}$ such that $(\widehat{\phi}, u) \in L^{\phi}$ for some $u, L^{\phi}=L^{\widehat{\phi}}$. For disjoint sets $\phi, \psi \subset \Phi_{t}$, for each $u$ the sets $\widehat{\phi}$ and $\widehat{\psi}$ such that $(\widehat{\phi}, u) \in L^{\phi}$ and $(\widehat{\psi}, u) \in L^{\psi}$ are disjoint.

Proof. For $\phi \subset \Phi_{t}$ there is a unique set $\widehat{\phi}=L(t, \phi, u)$ such that $(\widehat{\phi}, u) \in L^{\phi}$. The first statement follows from the transitivity property L2. The second property follows from L1.

Lemma 1 states that each traffic set defines a unique trace and each trace determines at each time a unique traffic set. Furthermore disjoint traffic sets define traces that do not intersect at any time.

Lemma 1 offers a ground to define the following process based on a process of calls in the traffic. For a traffic set $\phi$, consider the process $\mathbf{C}_{\phi}=\left\{C_{\phi}(s) ; s \in \mathbb{R}\right\}$ counting the number of calls in trace $L^{\phi}: C_{\phi}(s)$ gives the number of active calls present in trace $L^{\phi}$ at time $s$. A call generated by a subscriber in traffic set $\phi \subset \Phi_{t}$ follows its path, i.e. at time $s>t$ it will be in $L(t, \phi, s)$ assuming its call length exceeds $s-t$. Thus, calls generated in trace $L^{\phi}$ stay in $L^{\phi}$ for the whole activity period. Furthermore, for disjoint sets $\phi, \psi \subset \Phi_{t}$, calls generated in $L^{\phi}$ never occur in $L^{\psi}$. Due to L3, the volume of traffic in trace $L^{\phi}$ is preserved throughout time. Therefore calls arrive to $L^{\phi}$ with Poisson rate

$$
\widehat{\lambda}_{\phi}=\alpha V(\phi)
$$

while the period of time a call stays in $L^{\phi}$ is drawn from the call length distribution $G$. These observations lead to the following results for the transient behavior of the process $\mathbf{C}_{\phi}$.

Theorem 2 Assume that time $t_{0}$ exists such that no active calls are present in $\Phi_{t_{0}}$. For $\phi \subset \Phi_{t}$ and all $s \geq t_{0}, C_{\phi}(s)$ is Poisson distributed with finite mean

$$
\mathbb{E}\left[C_{\phi}(s)\right]=\alpha V(\phi) \cdot\left(\mathbb{E}\left[S \mid S \leq s-t_{0}\right] G\left(s-t_{0}\right)+\left(s-t_{0}\right)\left(1-G\left(s-t_{0}\right)\right)\right) .
$$

Furthermore, for disjoint sets $\phi, \psi \subset \Phi_{t}$, the processes $\mathbf{C}_{\phi}$ and $\mathbf{C}_{\psi}$ are independent. 
Proof. The process $\mathbf{C}_{\phi}$ is stochastically equivalent to a process counting the number of busy servers in an $M / G / \infty$ queue with arrival rate $\widehat{\lambda}_{\phi}=\alpha V(\phi)$ and i.i.d. service time distribution $G$ which implies the Poisson distribution of $C_{\phi}(s)$, and

$$
\mathbb{E}\left[C_{\phi}(s)\right]=\mathbb{E}\left[\int_{\max \left\{s-S, t_{0}\right\}}^{s} \widehat{\lambda}_{\phi} d u\right]=\widehat{\lambda}_{\phi} \mathbb{E}\left[s-\max \left\{s-S, t_{0}\right\}\right]
$$

(see e.g. Massey \& Whitt (1993) and Keilson \& Servi (1994)), hence (2). Lemma 1 argues that for any times $s$ and $u, C_{\phi}(s)$ and $C_{\psi}(u)$ are independent for disjoint $\phi, \psi \subset \Phi_{t}$, which implies that the processes $\mathbf{C}_{\phi}$ and $\mathbf{C}_{\psi}$ are independent.

Remark 3 (Initial conditions) The restrictions on the initial condition in Theorem 2 can be relaxed, see e.g. Keilson \& Servi (1994). For example, if at time $t_{0}$ a Poisson random number of calls are generated uniformly over traffic $\Phi_{t_{0}}$ then Theorem 2 still holds true with an additional term in (2) depending on the intensity of initial distribution.

Remark 4 (Time dependent fresh call arrivals) Call generation characteristics can readily be extended to a non-stationary setting as is the case when observing daily behaviour of subscribers. Subscribers generate less calls during early morning hours than during the traffic peak hours. To include this behaviour into our setting, consider a time dependent arrival rate of fresh calls per unit mass of subscribers $\alpha(t)$. For any traffic set $\phi$, calls arrive to $L^{\phi}$ with Poisson rate

$$
\widehat{\lambda}_{\phi}(t)=\alpha(t) V(\phi) .
$$

The process $C_{\phi}$ is stochastically equivalent to a process counting the number of busy servers in an $M_{t} / G / \infty$ queue with arrival rate $\widehat{\lambda}_{\phi}(t)$ and i.i.d. service time distribution $G$ which again implies a Poisson distribution for $C_{\phi}(s)$ with

$$
\mathbb{E}\left[C_{\phi}(s)\right]=\mathbb{E}\left[\int_{\max \left\{s-S, t_{0}\right\}}^{s} \widehat{\lambda}_{\phi}(u) d u\right],
$$

assuming no calls are present in traffic at time $t_{0}$.

An important special case is $t_{0} \rightarrow-\infty$, indicating that the system has been in operation long enough for the call characteristics to have reached equilibrium. We assume this is the case from this point on. Hence, for $\phi \subset \Phi_{t}$ and all $s, C_{\phi}(s)$ is Poisson distributed with finite mean

$$
\mathbb{E}\left[C_{\phi}(s)\right]=\alpha V(\phi) \mathbb{E}[S] .
$$

While the call behaviour in traces under the assumptions above is stationary, the mobility characteristics of the subscribers make the behaviour of the load offered to the cells time dependent. This behaviour is studied below.

Consider cell $A \subset \Omega$. Let $\mathbf{D}_{A}=\left\{D_{A}(s) ; s \in \mathbb{R}\right\}$ be the process counting the number of active calls in cell $A$. The arrival rate of calls to cell $A$ consists of fresh call arrivals with time varying arrival rate $\lambda_{A}(t)$ of (1) and of handovers migrating 
into cell $A$ from neighbouring cells due to the mobility of subscribers following the flow function $L$. The distribution of $D_{A}(t)$ can now be obtained from the number of calls in the trace of $A_{t}$ and the corresponding process $\mathbf{C}_{A_{t}}$ as is apparent from relation

$$
D_{A}(t)=C_{A_{t}}(t)
$$

Corollary 5 For any positive integer $k$ and any disjoint cells $A^{1}, \ldots, A^{k}$, the random variables $D_{A^{1}}(t), \ldots, D_{A^{k}}(t)$ are independent and Poisson distributed with finite means

$$
\mathbb{E}\left[D_{A^{i}}(t)\right]=\lambda_{A^{i}}(t) \mathbb{E}[S], \quad i=1, \ldots, k, t \in \mathbb{R}
$$

Proof. For disjoint cells $A^{1}, \ldots, A^{k}$ the corresponding traffic sets $A_{t}^{1}, \ldots, A_{t}^{k}$ at time $t$ are disjoint as well. Theorem 2 implies that the distributions of $D_{A^{i}}(t)$, $i=1, \ldots, k$, are independent Poisson with means $\lambda_{A^{i}}(t) \mathbb{E}[S]$.

Observe that while for disjoint cells $A, B \subset \Omega$, the numbers of calls $D_{A}(t)$ and $D_{B}(t)$ are independent for a fixed $t$, the processes $\mathbf{D}_{A}$ and $\mathbf{D}_{B}$ are generally not independent.

The model studied in this section is a special case of the Poisson-arrival-location model (PALM) introduced in Massey \& Whitt (1993): a Poisson random measure on $\mathbb{R} \times \Phi_{0} \times \mathbb{R}$ is determined by the arrival rate $\lambda$, homogeneity of calls over the traffic and the call length distribution. While Massey \& Whitt study the distribution of the number of calls in a cell of the wireless network with call holding time distribution related only to the cells (the classical queueing network approach), the special setting of our model enables us to study the influence of the call length distribution. In particular, as an immediate consequence of our modelling approach, we can conclude that when calls are homogeneously generated in traffic and subsequently follow the flow of traffic then the numbers of calls in disjoint traffic sets (or cells) are independent (at fixed time) and Poisson distributed with rate proportional to the mass of traffic in the sets, depending on the call length distribution only through its mean.

Remark 6 (Blocking of calls) The model analyzed above does not include blocking of calls such as naturally occurring in wireless networks due to their capacity restrictions. Our model is a step towards obtaining blocking probabilities for such networks that take into account time-varying rates due to customer mobility, and general call lengths. For example, the number of calls in the cells of our network with unlimited capacity is an upper bound on that number in cells with finite capacity, where blocked calls are cleared from the network, see e.g. Stoyan (1983). Alternatively, approximations such as the modified offered load approximation, and the pointwise stationary approximation might be generalized to take into account the general call length distribution. These approximations use the relation in equilibrium between the infinite server queue and the Erlang loss queue as the basis for the approximation of the network of Erlang loss queues with time-varying rates via a truncation of the state distribution of the network with unlimited capacity. For details see Massey \& Whitt (1993). 


\section{Examples and extensions}

Section 3.1 demonstrates that the traffic flow model incorporates most of the standard traffic models and Section 3.2 considers superposition of flows. Finally, Section 3.3 extends the result to a queueing network setting with random holding times in the queues, which allows us to model road traffic systems with random fluctuations in the velocity of subscribers.

\subsection{Standard highway model}

Consider a single lane road with cars that cannot overtake, see e.g. Lighthill \& Whitham (1955) and Newell (1993) for a complete description. For some reference car let $A(x, t)$ be the cumulative number of vehicles to pass some location $x$ by time $t$ starting from the passage of some reference car. Then, smoothing the curve of $A(x, t)$ such that it is twice differentiable, $A(x, t)$ determines the flow of traffic $q(x, t)$ through location $x$ at time $t$, and the density of traffic $k(x, t)$ on location $x$ at time $t$ :

$$
q(x, t)=\frac{\partial A(x, t)}{\partial t}, \quad k(x, t)=-\frac{\partial A(x, t)}{\partial x} .
$$

The velocity $v(x, t)$ on location $x$ at time $t$ is obtained from

$$
q(x, t)=k(x, t) v(x, t)
$$

while differentiation of $A$ yields the traffic mass conservation principle when there are no external departing or arriving flows,

$$
\frac{\partial k(x, t)}{\partial t}+\frac{\partial q(x, t)}{\partial x}=0
$$

For a subscriber on location $x$ at time $t$, its trajectory $\chi(t, x, \cdot)$ is determined by a solution of the differential equation

$$
\frac{d y}{d u}=v(y, u), \quad y(t)=x .
$$

Assuming $v$ is continuous and differentiable, there is a unique solution for any pair $(x, t) \in \Omega \times \mathbb{R}$. Hence for any times $t$ and $u, \chi(t, \cdot, u)$ is bijective and transitive. The mass preservation property

$$
\int_{U} k(x, t) d x=\int_{\chi(t, U, u)} k(y, u) d y
$$

for any cell $U \subset \mathbb{R}$ and any times $t, u$ can be shown by applying conservation principle (4). The flow function $L_{\chi}$ corresponding to this setting is

$$
L_{\chi}(t,(x, h), u):=\left(\chi(t, x, u), h \cdot \frac{k(\chi(t, x, u), u)}{k(x, t)}\right),
$$

for any times $u, t \in \mathbb{R}$, and any position $(x, h) \in \Phi_{t}$, i.e. any location $x \in \Omega$ and any $h$ with $0 \leq h \leq k(x, t)$. It can readily be shown that this flow function satisfies L1, L2 and L3. 


\section{Location independent velocity field}

Consider the special case with all subscribers travelling at equal velocity, $v(x, t) \equiv$ $v(t), t \in \mathbb{R}$. Then

$$
\chi(t, x, u):=x+\int_{t}^{u} v(s) d s .
$$

The density now satisfies the relation

$$
k(x, u)=k\left(x-\int_{t}^{u} v(s) d s, t\right) .
$$

The corresponding flow function is of a similar simple form,

$$
L_{\chi}(t,(x, h), u):=\left(x+\int_{t}^{u} v(s) d s, h\right),
$$

for any $u, t \in \mathbb{R}$, and any position $(x, h) \in \Phi_{t}$.

\subsection{Superposition of flows}

Consider two independent traffic streams on the same location space $\Omega$ with densities $k^{1}(x, t)$ and $k^{2}(x, t)$ and flow functions $L^{1}$ and $L^{2}$. The total traffic is then obtained as the superposition of these flows. The joint traffic flow is determined by the joint traffic density $k(x, t)=k^{1}(x, t)+k^{2}(x, t)$, and the joint flow function $L$ can be defined as

$$
L(t,(x, h), u):=\left\{\begin{array}{cc}
L^{1}(t,(x, h), u) & h \leq k^{1}(x, t), \\
L^{2}\left(t,\left(x, h-k^{1}(x, t)\right), u\right)+\left(0, k^{1}(x, u)\right) & h>k^{1}(x, t),
\end{array}\right.
$$

for any times $u, t \in \mathbb{R}$, and any position $(x, h) \in \Phi_{t}$.

Flows on multi-lane roads, two-way highways and road junctions can be straightforwardly modelled by a superposition of flows. A single car can be described by a single flow with density having a limited support that keeps its shape throughout time. A superposition of a number of such traffic flows would describe traffic in detail. Further examples of a superposition of flows extend the model to general traffic networks. Another extension incorporating nondeterministic holding times is the queueing network in Section 3.3 below.

\subsection{Networks of infinite server queues}

This section considers networks of infinite server queues with customers that have service times extending over multiple queues. For simplicity of notation we will restrict our attention to the case of exponential holding times in the queues, but general call length distribution.

Consider a tandem network of infinite server queues, labelled $j=\ldots,-1,0,1, \ldots$. Customers arrive to queue $j$ according to a non-homogeneous Poisson arrival process with arrival rate $\lambda_{j}(t)$. Assume that the arrival rate $\lambda_{j}(t)$ of customers is determined by a virtual traffic flow of subscribers moving among the queues. If this virtual flow 
travels with rate $\mu_{j}$ through queue $j$, then the arrival rate of customers in queue $j$ changes according to

$$
\frac{d \lambda_{j}(t)}{d t}=\mu_{j-1} \lambda_{j-1}(t)-\mu_{j} \lambda_{j}(t)
$$

We say that the queueing network admits service mass preservation ( $S M P$ ) when (5) holds for each queue $j$ and each time $t$.

The holding times $H_{j}$ of customers in queue $j$ are i.i.d. exponentially distributed with mean $\mu_{j}^{-1}$ (naturally corresponding to the movement of the virtual traffic flow), independent of holding times in other queues and of the arrival process. The service requests $S$ of customers extend over multiple queues and are i.i.d. with general distribution $G$, independent of the arrival process and of holding times in the queues: a customer stays in the network until his service is over, but might traverse a number of queues during his stay. A customer in queue $j$ leaves this queue when either

1. his remaining service time is shorter than his holding time (he leaves the system at the end of his service), or

2. his holding time is shorter than his remaining service time (he routes to queue $j+1$, there drawing a new exponentially distributed holding time with mean $\left.\mu_{j+1}^{-1}\right)$.

Theorem 7 shows that the queue length process in the queueing network under SMP with random holding times in the queues satisfies a relation similar to that obtained in Corollary 5 for the cellular network. To this end, let $K_{j}(t)$ denote the number of customers in queue $j$ at time $t$. Assume that $\mu_{j}^{-1} \geq \epsilon>0$ for all $j$, and that $\lambda_{j}(t) \leq M$ for all $j$ and all $t$. For simplicity we assume the network started empty at $t=-\infty$.

Theorem 7 Consider the queueing network above satisfying service mass preservation (5). The distribution of number of customers in queue $j$ at time $t$ is Poisson with mean

$$
\mathbb{E}\left[K_{j}(t)\right]=\lambda_{j}(t) \mathbb{E}[S]
$$

Proof. As the arrival process is Poisson with rates in (5) and customers move independently the analysis of Massey \& Whitt (1993) applies and it remains to compute the mean $\mathbb{E}\left[K_{j}(t)\right]$. An explicit expression will be obtained similar to the proof of Corollary 5 by backward tracing of customers.

Let $H_{j}^{n}$ denote the total random holding time of a customer from queue $j-n$ up to queue $j-1$ :

$$
H_{j}^{n}:=\sum_{i=j-n}^{j-1} H_{i},
$$

with distribution $F_{j}^{n}(y)$ and $H_{j}^{0}=0$. Let $P_{j}^{n}(y)$ denote the probability that a customer originating in queue $j-n$ at some time $u$ will be in queue $j$ at time $u+y$ providing it has not yet terminated, that is

$$
P_{j}^{n}(y):=\mathbb{P}\left[H_{j}^{n}+H_{j} \geq y, H_{j}^{n} \leq y\right]=F_{j}^{n}(y)-F_{j+1}^{n+1}(y),
$$


where $P_{j}^{0}(y)=1-F_{j}(y)$, and $P_{j}^{-1}(y)=0$. Notice that $P_{j}^{n}(0)=0, n \geq 1$, and $P_{j}^{0}(0)=1$. $F_{j}^{n}(y)$ and $P_{j}^{n}(y)$ are continuous and continuously differentiable on $[0, \infty)$

We will first show that for each $j$ and $n \geq 0$,

$$
\mu_{j-n} P_{j}^{n}(y)-\mu_{j-n} P_{j}^{n-1}(y)+\frac{d}{d y} P_{j}^{n}(y)=0 .
$$

This is clear for $n=0$. For $n \geq 1$, let $\varphi_{j}^{n}(s)=\mathbb{E}\left[e^{-s H_{j}^{n}}\right]$, which exists for $\operatorname{Re}(s) \geq 0$. As $H_{j}^{n}$ is a sum of independent exponentially distributed random variables,

$$
\varphi_{j}^{n}(s)=\prod_{i=j-n}^{j-1} \frac{\mu_{i}}{s+\mu_{i}}, \quad \operatorname{Re}(s) \geq 0 .
$$

Taking the Laplace-Stieltjes transform in (7), using (6), we arrive at

$$
\mu_{j-n}\left(\varphi_{j}^{n}(s)-\varphi_{j+1}^{n+1}(s)\right)-\mu_{j-n}\left(\varphi_{j}^{n-1}(s)-\varphi_{j+1}^{n}(s)\right)+s\left(\varphi_{j}^{n}(s)-\varphi_{j+1}^{n+1}(s)\right)=0,
$$

for all $s, \operatorname{Re}(s) \geq 0$. Inserting (8) into this expression allows us to conclude (7).

Now consider the arrival rate of customers from other queues into queue $j$,

$$
\nu_{j}(t, u):=\sum_{n=0}^{\infty} \lambda_{j-n}(t-u) P_{j}^{n}(u),
$$

for some $j$ and $t$, which is clearly finite since $\lambda_{j}(t-u) \leq M$ for every $j$ for each $u$. The rate $\nu_{j}(t, u)$ is constant in $u$. To this end, observe that, using (5),

$$
\begin{aligned}
\frac{d}{d u} \nu_{j}(t, u)= & \sum_{n=0}^{\infty}\left[-\mu_{j-n-1} \lambda_{j-n-1}(t-u)+\mu_{j-n} \lambda_{j-n}(t-u)\right] \cdot P_{j}^{n}(u)+ \\
& +\sum_{n=0}^{\infty} \lambda_{j-n}(t-u) \cdot \frac{d}{d u} P_{j}^{n}(u) \\
= & \sum_{n=0}^{\infty} \lambda_{j-n}(t-u)\left[\mu_{j-n} P_{j}^{n}(u)-\mu_{j-n} P_{j}^{n-1}(u)+\frac{d}{d u} P_{j}^{n}(u)\right]=0,
\end{aligned}
$$

where the last equality follows from (7) and we have used that all the sums are uniformly convergent, since $\mu_{j} \leq \epsilon$ for each $i$. Thus, for all $j$ and any $u, t$,

$$
\sum_{n=0}^{\infty} \lambda_{j-n}(t-u) P_{j}^{n}(u)=\nu_{j}(t, 0)=\lambda_{j}(t) .
$$

We are now ready to calculate $\mathbb{E}\left[K_{j}(t)\right]$. With a random service time extending over multiple queues, $\mathbb{P}\left[H_{j}^{n}+H_{j} \geq u, H_{j}^{n} \leq u, S \geq u\right]$ gives the probability that a customer arriving to the system in queue $j-n$ is present in queue $j$ after the time period of $u$. Therefore, the expected number of customers arriving to the system in queue $j-n$ and present in cell $j$ at time $t$ is

$$
\begin{aligned}
\int_{0}^{\infty} \lambda_{j-n}(t-u) \mathbb{P}\left[H_{j}^{n}+H_{j}\right. & \left.\geq u, H_{j}^{n} \leq u, S \geq u\right] d u= \\
& =\int_{0}^{\infty} \lambda_{j-n}(t-u) P_{j}^{n}(u) \mathbb{P}[S \geq u] d u .
\end{aligned}
$$


Summing this expression over all $n \geq 0$ yields the expected number of customers in queue $j$ at time $t$. Thus, using (9),

$$
\begin{aligned}
\mathbb{E}\left[K_{j}(t)\right] & =\sum_{n=0}^{\infty} \int_{0}^{\infty} \lambda_{j-n}(t-u) P_{j}^{n}(u) \mathbb{P}[S \geq u] d u \\
& =\lambda_{j}(t) \int_{0}^{\infty} \mathbb{P}[S \geq u] d u=\lambda_{j}(t) \mathbb{E}[S]
\end{aligned}
$$

The result of Theorem 7 can readily be extended to a network of $N$ infinite server queues labelled $j=1,2, \ldots, N$, with exponential holding times $H_{j}$ with mean $\mu_{j}^{-1}$ in queue $j$ and general service time $S$ extending over multiple queues where arrival process, holding times and service times are independent. A customer leaving queue $j$ with positive remaining service time routes to queue $i$ with probability $p_{j i}$ in accordance with the mean virtual traffic flow, where $\sum_{i=1}^{N} p_{j i}=1$. A customer leaving queue $j$ due to completition of his service leaves the network.

Service mass preservation is expressed as

$$
\frac{d \lambda_{j}(t)}{d t}=\sum_{i=1}^{N} \mu_{i} \lambda_{i}(t) p_{i j}-\mu_{j} \lambda_{j}(t), \quad j=1, \ldots, N .
$$

Theorem 7 applies for any deterministic path through this network. Superposition of different paths can be extended to this setting which allows us to conclude the following result.

Theorem 8 Consider the queueing network satisfying service mass preservation (10). The distribution of number of customers in queue $j$ at time $t$ is Poisson with mean

$$
\mathbb{E}\left[K_{j}(t)\right]=\lambda_{j}(t) \mathbb{E}[S]
$$

Remark 9 Insensitivity results for infinite server queues in equilibrium are wellestablished. In contrast, the queue length distribution of infinite server queues with time-varying arrival rates depends in general on the service time distribution through all its moments, see e.g. Massey \& Whitt (1993). Theorems 7 and 8 provide insensitivity results for a network with time-varying arrival rates. These results are due to the service mass preservation properties (5) and (10) describing the movement of subscribers among the queues. Service mass preservation mimics the properties of the traffic flow function, that allow for a transformation of the network with timevarying rates into a stationary setting, which shows that our network with timevarying arrival rates operates on the edge between transient networks and networks in equilibrium.

\section{Conclusion}

This paper has developed a modelling approach for wireless networks with subscriber movement driven by a fluid traffic that includes standard road traffic models. 
Through a transformation of the network characteristics to properties of the traffic sets, the distribution of calls over the cells of the network is shown to be a multi dimensional Poisson distribution with time-varying mean that depends on the call length distribution (that extends over multiple cells) only through its mean. This result ignores capacity constraints and blocking of calls, and is a step towards developing offered load approximations for wireless networks with time-varying arrival rates (such as e.g. due to a traffic jam moving along a road), and general call length distribution, which is our aim for further research.

\section{References}

Boucherie, R. J. \& Van Dijk, N. M. (2000), 'On a queueing network model for cellular mobile telecommunications networks', Operations Research 48, 38-49.

Everitt, D. (1994), 'Traffic engineering of the radio interface for cellular mobile networks', Proceedings of the IEEE 82, 1371-1382.

Keilson, L. \& Servi, L. D. (1994), 'Networks of non-homogeneous $M / G / \infty$ systems', Journal of Applied Probability 31A, 157-168.

Leung, K. K., Massey, W. A. \& Whitt, W. (1994), 'Traffic models for wireless communication networks', IEEE Journal on Selected Areas in Communications 12, 1353-1364.

Lighthill, M. J. \& Whitham, G. B. (1955), 'On kinematic waves. II: A theory of traffic flow on long crowded roads', Proceedings of The Royal Society A229, 317-345.

Massey, W. A. \& Whitt, W. (1993), 'Networks of infinite-server queues with nonstationary poisson input', Queueing Systems 13, 183-250.

Massey, W. A. \& Whitt, W. (1994), 'A stochastic model to capture space and time dynamics in wireless communication systems', Probability in the Engineering and Informational Sciences 8, 541-569.

Newell, G. F. (1993), 'A simplified theory of kinematic waves in highway traffic, part I: General theory', Transportation Research 27B, 281-287.

Stoyan, D. (1983), Comparison Methods for Queues and Other Stochastic Models, Wiley, Berlin. 